\title{
Regional White Matter Variation Associated with Domain-specific Metacognitive Accuracy
}

\author{
Benjamin Baird ${ }^{1}$, Matthew Cieslak ${ }^{1}$, Jonathan Smallwood ${ }^{2}$, \\ Scott T. Grafton ${ }^{1}$, and Jonathan W. Schooler ${ }^{1}$
}

\begin{abstract}
The neural mechanisms that mediate metacognitive ability (the capacity to accurately reflect on one's own cognition and experience) remain poorly understood. An important question is whether metacognitive capacity is a domain-general skill supported by a core neuroanatomical substrate or whether regionally specific neural structures underlie accurate reflection in different cognitive domains. Providing preliminary support for the latter possibility, recent findings have shown that individual differences in metacognitive ability in the domains of memory and perception are related to variation in distinct gray matter volume and resting-state functional connectivity. The current investigation sought to build on these findings by evaluating how metacognitive ability in these domains is related to variation in white matter microstructure. We quantified metacognitive ability across memory and perception domains and used
\end{abstract}

\section{INTRODUCTION}

Metacognition refers to reflection on or analysis of one's own cognitive processes. The ability "to doubt what one knows, to deny or affirm one's beliefs, to judge one's own memories and percepts, to comment on one's dreams, to recollect and reflect on one's own past" (Terrace \& Metcalf, 2004, p. 2) represent several of the core abilities commonly referred to under the umbrella term of metacognition (Metcalfe \& Shimamura, 1994). Metacognition may be viewed as a general category that encompasses these processes, as well as introspection, which has been proposed to be a special case of metacognition involving specifically conscious content as the object of reflection (Fleming, Dolan, \& Frith, 2012; Overgaard \& Sandberg, 2012). However, metacognition appears to be distinct from other potentially related constructs such as intelligence, as individual differences in metacognitive ability are uncorrelated with general fluid intelligence ( $g$; Fleming, Huijgen, \& Dolan, 2012).

Despite the seeming immediacy with which we reflect on our minds, a central insight that has emerged from research in the cognitive sciences over the past 40 years

\footnotetext{
${ }^{1}$ University of California, Santa Barbara, ${ }^{2}$ University of York
}

diffusion spectrum imaging to examine the relation between high-resolution measurements of white matter microstructure and individual differences in metacognitive accuracy in each domain. We found that metacognitive accuracy for perceptual decisions and memory were uncorrelated across individuals and that metacognitive accuracy in each domain was related to variation in white matter microstructure in distinct brain areas. Metacognitive accuracy for perceptual decisions was associated with increased diffusion anisotropy in white matter underlying the ACC, whereas metacognitive accuracy for memory retrieval was associated with increased diffusion anisotropy in the white matter extending into the inferior parietal lobule. Together, these results extend previous findings linking metacognitive ability in the domains of perception and memory to variation in distinct brain structures and connections. is that our access to our minds is noisy and subject to inaccuracies and dissociations (e.g., Schooler \& Schreiber, 2004; Schooler, 2002). Metacognitive awareness is particularly compromised for causal-explanatory theorizing about the reasons for actions or decisions (Hall, Johansson, Tärning, Sikström, \& Deutgen, 2010; Johansson, Hall, Sikström, \& Olsson, 2005; Gazzaniga \& LeDoux, 1978; Nisbett \& Wilson, 1977). For example, classic studies revealed that individuals whose choice preferences are biased through priming or position effects are generally unaware of these effects and confabulate reasons for their selections (Nisbett \& Wilson, 1977). Other research has illustrated the frequent dissociations that occur in metacognitive monitoring of the ongoing state of one's mind. One striking (and perhaps relatable) example is that individuals often fail to notice that their minds have wandered to unrelated topics during reading or sustained attention tasks, even in the context of experiments in which they are specifically instructed to remain vigilant for such lapses and report them as soon as they occur (Schooler et al., 2011; Christoff, Gordon, Smallwood, Smith, \& Schooler, 2009; Smallwood, McSpadden, \& Schooler, 2008; Schooler, Reichle, \& Halpern, 2004).

The cognitive and neural mechanisms that mediate the fidelity of metacognitive awareness remain poorly 
understood. Recent research has exploited individual differences in metacognitive accuracy in healthy individuals as one approach to elucidating the neural mechanisms underlying the capacity to accurately reflect on particular cognitive processes (Baird, Smallwood, Gorgolewski, \& Margulies, 2013; McCurdy et al., 2013; Fleming, Weil, Nagy, Dolan, \& Rees, 2010). These studies converge with prior work in documenting a primary role of $\mathrm{pFC}$ in metacognition, particularly the anterior pFC (aPFC; see Fleming \& Dolan, 2012, for a review). However, it remains equivocal whether metacognitive ability is a domain-general skill supported by a single neuroanatomical substrate or whether it varies across different processes or cognitive domains. On the one hand, the fact that metacognition has been linked to a "higher-order" brain region in $\mathrm{pFC}$ might suggest that it is supported by brain mechanisms that supersede cognition-level processing in a given domain, supporting a domain-general rather than domain-specific account (Song et al., 2011). On the other hand, if metacognitive ability depends on the integration between $\mathrm{pFC}$ and cognition-level processing, it is also plausible that multiple brain networks linking domain-specific processing in posterior regions to frontal cortex could underlie metacognitive capacity in a particular domain (Shimamura, 2000; Nelson \& Narens, 1990).

Patient populations display greater impairment for some types of metacognitive tasks compared with others, potentially because they are supported by distinct neural structures. For instance, schizophrenic patients appear to have a relatively preserved capacity to make retrospective metacognitive judgments of their memory as well as trialby-trial judgments of their performance accuracy, despite showing significant impairment in metacognitive judgments of their own agency as well as a generalized impairment in insight into their disorder (David, Bedford, Wiffen, \& Gilleen, 2012; Metcalfe, Van Snellenberg, DeRosse, Balsam, \& Malhotra, 2012). Furthermore, preliminary studies in healthy individuals have suggested that there may be domain specificity in the neural basis of metacognitive ability in distinct cognitive domains. Specifically, two recent studies found that individuals' metacognitive accuracy in perception and memory tasks were related to differential neural substrates. McCurdy et al. (2013) found that gray matter volume in the distinct regions of the lateral aPFC and precuneus covaried with metacognitive accuracy in perception and memory domains, respectively. Furthermore, Baird et al. (2013) found that individual differences in metacognitive accuracy in each domain were associated with resting-state functional connectivity in distinct brain networks. Metacognitive accuracy for perceptual decisions was linked to greater connectivity between lateral aPFC and the right dorsal ACC (dACC) and dorsal striatum, whereas metacognitive accuracy for memory retrieval was related to greater connectivity between medial aPFC and the right precuneus and inferior parietal lobule (IPL).

In the current investigation, we sought to build on these findings by evaluating how metacognitive ability in percep- tual and mnemonic domains is related to individual differences in anatomical connectivity strength. We first quantified metacognitive accuracy in perception and memory domains and assessed intraindividual covariance in metacognitive accuracy. We then used diffusion spectrum imaging (DSI; Schmahmann et al., 2007; Wedeen, Hagmann, Tseng, Reese, \& Weisskoff, 2005) to examine the relation between high-resolution measurements of white matter microstructure and individual differences in metacognitive accuracy for perceptual decisions and mnemonic judgments. Most previous studies examining the relationship between cognitive abilities and variation in white matter anatomy have relied on the diffusion tensor model and specifically averaged fractional anisotropy (FA), which is limited in its ability to model the crossing-fiber architecture of white matter that is ubiquitous in the brain (Jones, Knösche, \& Turner, 2013). Specifically, diffusion tensor imaging (DTI) significantly underestimates the actual distribution of fiber pathways and can be inaccurate in regions of partial volumes of cerebrospinal fluid or gray matter (Vos, Jones, Viergever, \& Leemans, 2011; Oouchi et al., 2007; Alexander, Hasan, Lazar, Tsuruda, \& Parker, 2001). In the current study, we therefore used DSI with its much higher angular resolution to offset these problems, allowing us to examine the relation between highresolution measurements of white matter structure and individual differences in metacognitive ability. On the basis of the previous findings discussed above, we hypothesized that regional variation of white matter microstructure would underlie individual differences in metacognitive ability in each cognitive domain.

\section{METHODS \\ Participants}

Forty-two participants completed the experiment (20 men, age range $=18-47$ years, mean age $=21.5$ years). Four participants who completed the cognitive testing component were excluded from the brain imaging analysis: one participant because he was left-handed, one participant because of suboptimal quantitative anisotropy thresholding, and two participants because they had a metal oral appliance that created a large artifact in the DSI scan. Signed informed consent was obtained from all participants before completing the study, and ethical approval for the study was obtained from the University of California, Santa Barbara, institutional review board. All participants in the final sample $(n=38)$ were right-handed, had normal or corrected-to-normal vision, and had no history of neurological or psychiatric disease.

\section{Stimuli}

Stimuli and tasks were programmed in MATLAB version 7.9 (The Mathworks Inc., Natick, MA) using the Psychophysics Toolbox version 3.0 (Kleiner et al., 2007; Brainard, 
1997). Stimuli for the perceptual decision task consisted of visual displays composed of six Gabor gratings arranged in a circle around a fixation point at an eccentricity of 6.5 visual degrees (Figure 1A). Each grating subtended 2.8 visual degrees and consisted of vertical alternating light and dark bars modulated at a spatial frequency of 2.2 cycles per visual degree at a contrast of $20 \%$. Stimuli were presented in a darkened room at a viewing distance of approximately $60 \mathrm{~cm}$.

Stimuli for the memory retrieval task consisted of 320 neutral-valence noncomposite nouns selected from the Medical Research Council Psycholinguistic database (Wilson, 1988). All stimuli were five characters in length and had a word frequency between 1 and 800 per million.

\section{Tasks and Procedure}

Participants performed two experimental sessions: an MRI session in which DSI scans were acquired and a behavioral session in which they were asked to make metacognitive evaluations of perceptual decisions and mnemonic judgments. A schematic outline of the metacognitive tasks is shown in Figure 1. Task order was counterbalanced across participants.
The perceptual task was adapted from Song et al. (2011) and Fleming et al. (2010). Each trial $(n=320)$ consisted of a presentation of a 250-msec visual stimulus display consisting of six Gabor gratings arranged in a circle around central fixation, followed by an ISI of $500 \mathrm{msec}$ during which only the fixation cross remained on the screen, followed by a second 250-msec stimulus display consisting of six Gabors arranged around fixation (Figure $1 \mathrm{~A})$. In one of the two stimulus displays, the orientation of one of the Gabor patches was tilted slightly from the vertical axis. The display interval in which this "pop-out" Gabor occurred as well as its spatial location on the screen varied randomly across trials. The orientation tilt of the pop-out Gabor was adjusted using a 2-up 1-down adaptive staircase procedure (Fleming et al., 2010; Levitt, 1971) designed to result in a convergence on $70 \%$ accuracy for individual performance. Two consecutive correct responses resulted in a reduction of the orientation parameter by one step $\left(0.25^{\circ}\right)$, whereas one incorrect response resulted in an increase of the orientation parameter by one step. Following the offset of the second stimulus presentation, participants made unspeeded 2-choice discriminations as to whether the pop-out Gabor occurred in either the first or second stimulus display. Participants then rated their confidence in the accuracy of their response on a scale of

A Perceptual Decision Task: Schematic of Single Trial $(n=320)$
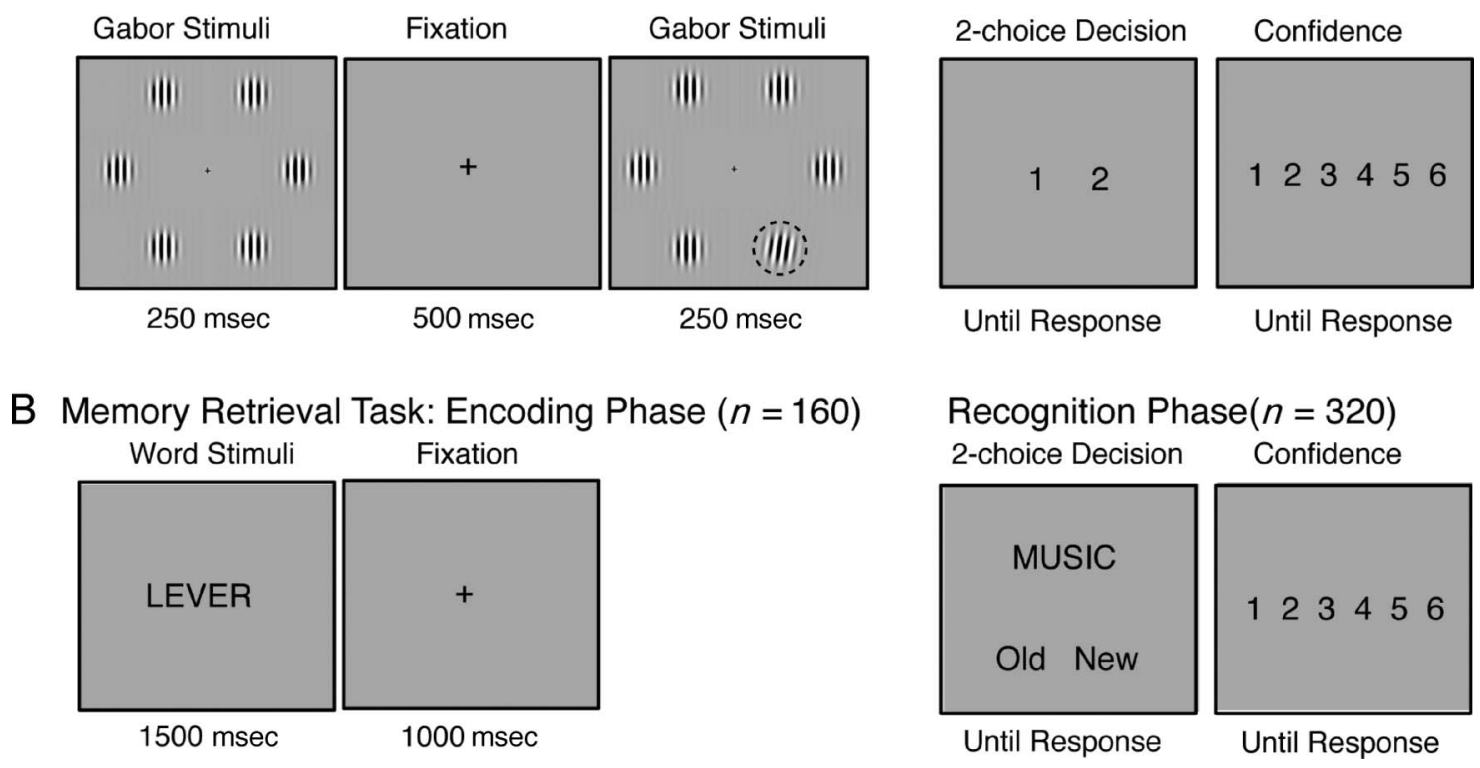

Figure 1. Experimental paradigm. Participants completed two tasks in a counterbalanced order. (A) Perceptual discrimination task. Each trial $(n=320)$ consisted of a visual display of six Gabor gratings, followed by an ISI of 500 msec, followed by a second visual display of six Gabor gratings. In one of the two displays, the orientation of one randomly selected Gabor patch was tilted slightly from the vertical axis (indicated here with a dashed circle that was not present in the actual display). The orientation angle of this pop-out Gabor was adjusted using a 2-up 1-down adaptive staircase procedure. Participants made unspeeded 2-choice discrimination judgments as to whether the "pop-out" Gabor occurred in either the first or second stimulus display and then rated their confidence in the accuracy of their response on a scale of 1 (low confidence) to 6 (bigh confidence). (B) Memory retrieval task. The memory task consisted of a classic verbal recognition memory paradigm. During encoding, participants viewed 160 words randomly selected from a set of 320 words. During recognition, participants were presented with each word from the full list of stimuli in a random order (half of which were presented during encoding and half of which were new) and were asked to make unspeeded 2-choice discrimination judgments as to whether the stimulus was old or new and then rated their confidence in their response. 
1 (low confidence) to 6 (high confidence; Fleming et al., 2010). All responses were made using the number pad on the keyboard.

The memory task consisted of two phases: encoding and recognition. Before beginning the encoding phase, participants were informed that a recognition phase would follow in which their memory for the presented words would be tested. During encoding, participants viewed 160 words randomly selected from the full set of 320 words presented sequentially in the center of the screen. Words were displayed for $1500 \mathrm{msec}$ and were separated by an ISI of $1000 \mathrm{msec}$ in which a fixation cross was displayed. During recognition, participants were presented with each word from the full list of stimuli in a random order (half of which were presented during encoding and half of which were new) and were asked to make unspeeded 2-choice discriminations as to whether the stimulus was old or new. Participants then rated their confidence in the accuracy of their response on a scale of 1 (low confidence) to 6 (high confidence). All responses were made using the number pad on the keyboard.

\section{Quantification of Metacognitive Ability}

Signal detection theory (SDT; Green \& Swets, 1966) was used to compute estimates of metacognitive accuracy, here quantified as the ability of an individual to discriminate between their own correct and incorrect perceptual decisions or mnemonic judgments with confidence ratings on a trial-by-trial basis. A primary concern in any metacognitive (Type II) analysis is to separate estimates of Type II sensitivity from the potential confounding influence of sensitivity on the primary (Type I) task (e.g., Galvin, Podd, Drga, \& Whitmore, 2003). Type II sensitivity refers to an individual's ability to discriminate between their own correct and incorrect responses, whereas Type I sensitivity refers to an individual's ability to discriminate between stimulus alternatives (i.e., their capacity to distinguish old items from new items in a recognition memory task; Higham, Perfect, \& Bruno, 2009; Clarke, Birdsall, \& Tanner, 1959). SDT approaches can quantify metacognitive accuracy independent of an observer's decision strategy or cognitive ability on the primary task, which have been shown to confound other methods of estimating metacognitive ability (Fleming \& Lau, 2014; Maniscalco \& Lau, 2012).

Metacognitive accuracy on the perceptual task was quantified using the computational methods outlined in Fleming et al. (2010). Because performance on the perceptual task is held constant with an online thresholding procedure, it is possible to compute a measure of metacognitive accuracy that is unconfounded by Type I performance directly from the empirical Type II receiver operating characteristic (ROC) curve. The Type II ROC curve reflects the relationship between the accuracy of visual discriminations and an observer's confidence rat- ings. To plot the ROC, $p$ (confidence $=i \mid$ correct) and $p$ (confidence $=i \mid$ incorrect) were calculated for each level of confidence $i$, transformed into cumulative probabilities and used to construct each $x, y$ point on the empirical ROC curve (Fleming et al., 2010; Kornbrot, 2006; Galvin et al., 2003). ROC curves were anchored at $[0,0]$ and $[1,1]$. The Type II ROC curve thus reflects the probability of being correct for each level of confidence. An ROC curve that rises steeply off the diagonal axis indicates that the likelihood of being correct increases with increasing confidence level, whereas a flat ROC along the major diagonal indicates a weak relationship between confidence and accuracy. When several points on the Type II ROC are available, an empirical estimate of the area under the ROC may be obtained, yielding a nonparametric measure of Type II sensitivity (Kornbrot, 2006). The area under the Type II ROC curve $\left(A_{\text {roc }}\right)$ when performance is held constant provides a robust estimate of metacognitive discrimination that is independent of Type I sensitivity. Type I sensitivity $\left(d^{\prime}\right)$ was calculated as $d^{\prime}=z(H)-z(\mathrm{FA})$, where $z$ represents the inverse of the cumulative normal distribution and $H=p$ (response $=1 \mid$ interval $=1)$ and $\mathrm{FA}=p($ response $=1 \mid$ interval $=2)$.

Quantification of metacognitive accuracy in the memory task required a computational approach that explicitly accounts for Type I performance. A model-based SDT approach to account for variance in primary task performance in the computation of Type II sensitivity has recently been described and validated (McCurdy et al., 2013; Maniscalco \& Lau, 2012). This method has been discussed at length elsewhere (Maniscalco \& Lau, 2012). Briefly, the approach exploits the link between Type I and Type II SDT models to express observed Type II sensitivity at the level of the Type I SDT model (termed meta $d^{\prime}$ ). Maximum likelihood estimation is used to determine the parameter values of the Type I SDT model that provide the best fit to the observed Type II data. A measure of metacognitive ability that controls for differences in Type I sensitivity is then calculated by taking the ratio of meta $d^{\prime}$ and the Type I sensitivity parameter $d^{\prime}: M_{\text {ratio }}=$ meta $d^{\prime} / d^{\prime}$. The most straightforward approach to computing $M_{\text {ratio }}$ involves an equal variance SDT model in which the variances of internal distributions of evidence for categorizing an item as "old" or "new" in the Type I model are assumed to be equal. However, this assumption is violated for 2-choice old/new recognition memory tasks (Mickes, Wixted, \& Wais, 2007; Swets, 1986). We therefore computed $M_{\text {ratio }}$ under an unequal variance SDT model, which uses the slope of the Type I zROC to infer the ratio of the standard deviations of the Type I distributions (s) underlying the two response categories and then holds this parameter constant in the estimation

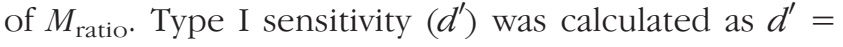
$z(H)-z(\mathrm{FA})$, where $z$ represents the inverse of the cumulative normal distribution and $H=p$ (response $=$ old $\mid$ stimulus $=$ old $)$ and $\mathrm{FA}=p($ response $=$ old $\mid$ stimulus $=$ new $)$. 


\section{MRI Acquisition}

DSI and T1-weighted anatomical scans were collected on a 3.0-T Siemens Tim Trio scanner equipped with highperformance gradients at the University of California, Santa Barbara, Brain Imaging Center. DSI scans sampled 257 directions with a maximum $b$ value of 5000 and an isotropic voxel size of $2.4 \mathrm{~mm}$ (axial acquisition, $1 \mathrm{~b} 0$ image, repetition time $=11.4 \mathrm{sec}$, echo time $=138 \mathrm{msec}$, 51 slices, field of view $=231 \times 231 \times 123 \mathrm{~mm}$ ). Before the diffusion-weighted scan, a high-resolution T1-weighted structural image was acquired using an MP-RAGE pulse sequence (repetition time $=2300 \mathrm{msec}$, echo time $=$ $2.98 \mathrm{msec}$, flip angle $=9^{\circ}$, field of view $=256 \mathrm{~mm}$, acquisition voxel size $=1 \times 1 \times 1.1 \mathrm{~mm}$ )

\section{DSI Data Processing}

DSI data were reconstructed in DSI Studio (www.dsi-studio. labsolver.org) using q-space diffeomorphic reconstruction (QSDR; Yeh \& Tseng, 2011). This technique first nonlinearly spatially normalizes an individual's DSI data and reconstructs spin density functions (SDFs) in standard space. Normalization was performed by registering individual anisotropy maps to the fMRI of the brain (FMIRB) $1 \mathrm{~mm}$ template (FSL, Oxford, UK) using a nonlinear registration implemented in DSI Studio (Ashburner \& Friston, 1999). Goodness-of-fit was assessed evaluating the $R^{2}$ statistic between the warped image and the template image (Yeh, Tang, \& Tseng, 2013). All participants had $R^{2}$ above .6, indicating good registration accuracy. QSDR on DSI data are able to reconstruct many complex fiber tract configurations, including crossing fibers. QSDR parameters were mean diffusion distance of $1.25 \mathrm{~mm}$ and three fiber orientations per voxel.

Deterministic fiber tracking was performed identically to Cieslak and Grafton (2014) using DSI Studio. The parameters included an angular cutoff of $55^{\circ}$, step size of $1.0 \mathrm{~mm}$, minimum length of $10 \mathrm{~mm}$, smoothing of $0.0 \mathrm{~mm}$, maximum length of $400 \mathrm{~mm}$, and a quantitative anisotropy threshold determined by DWI signal in the CSF. Tracking with a modified FACT algorithm was performed until 100,000 streamlines were reconstructed for each individual. Streamlines were labeled according to which the pair of regions in which they terminated. If a streamline did not intersect a labeled voxel within $5 \mathrm{~mm}$ of its endpoint, then the streamline was not considered for analysis.

\section{Structural (T1) Data Processing}

Cortical surface reconstruction was performed on T1 scans using FreeSurfer (Han et al., 2006; Jovicich et al., 2006; Fischl, Salat, et al., 2004; Fischl, van der Kouwe, et al., 2004; Segonne et al., 2004; Fischl et al., 2002; Fischl, Liu, \& Dale, 2001; Fischl \& Dale, 2000; Dale, Fischl, \& Sereno, 1999; Fischl, Sereno, \& Dale, 1999;
Fischl, Sereno, Tootell, \& Dale, 1999). Affine transformation from b0 space to T1 volume was calculated using Boundary-Based Registration (BBRegister; Greve \& Fischl, 2009). Anatomical scans were segmented using the connectome mapping toolkit (Hagmann et al., 2008). The Lausanne 2008 scale 33 (83 regions) atlas was registered and mapped to the b0 volume from each subject's DSI data. The b0 to Montreal Neurological Institute (MNI) voxel mapping produced via QSDR was then used to map region labels from native space to MNI coordinates. Regions were dilated by $4 \mathrm{~mm}$ in each direction to cover the gray/ white matter boundary. Dilation was performed identically to Cieslak and Grafton (2014).

\section{White Matter Microstructure (Diffusion Anisotropy) Data Processing}

To examine the relationship between metacognitive ability and white matter microstructure, we computed two high-resolution measures of diffusion anisotropy from DSI scans: generalized FA (GFA) and quantitative anisotropy (QA). These measures extend the FA measure from DTI, which has been used extensively as a measure of white matter microstructure, with changes due to either differences of myelination, axonal density, or degree of fiber crossing (e.g., Kraus et al., 2007; Kubicki et al., 2005). Lower FA values indicate that diffusion is more isotropic (i.e., undirected), whereas higher values indicate that diffusion has a stronger directional orientation. However, as noted above, FA is hindered by the substantial limitations of DTI reconstruction, particularly the partial volume effect (e.g., Barrick \& Clark, 2004). Specifically, estimates of FA are influenced by the presence of crossing fibers and partial volumes of other structures/tissues within a voxel such as CSF, which can lead to inaccurate measures of anisotropy (Vos et al., 2011; Oouchi et al., 2007; Alexander et al., 2001). GFA presents an extension of FA to high-angular resolution diffusion-weighted image that is capable of measuring anisotropy across multiple diffusion directions (Tuch, 2004). GFA is computed by dividing the standard deviation by the root mean square of the SDF. It thus reflects a similar measure of anisotropy to FA but is generalized across multiple fiber orientations (Cohen-Adad, Descoteaux, \& Wald, 2011). Unfortunately, GFA is also not totally immune from the partial volume effect (Yeh, Verstynen, Wang, Fernández-Miranda, \& Tseng, 2013; Fritzsche, Laun, Meinzer, \& Stieltjes, 2010). QA, on the other hand, reflects the anisotropy of the peak orientations of the SDF (Yeh, Wedeen, \& Tseng, 2010). QA is calculated by subtracting the background isotropic diffusion component from the SDF value at the resolved fiber orientation (Yeh et al., 2010). In the current study, we examined QA for the peak fiber orientation at each voxel. QA is less susceptible to the partial volume effect, but it is susceptible to other sources of MR acquisition noise (Yeh, Verstynen, et al., 2013). Each measure therefore has its relative strengths and both measures present an extension in 
high-resolution diffusion-weighted imaging to classical FA measures of white matter microstructure.

GFA and QA values were computed from QSDR reconstructed SDFs in MNI space. Because our previous investigation (Baird et al., 2013) found right lateralization to neural structures underlying metacognitive ability, we initially focused our analysis of the relationship between metacognitive ability and white matter microstructure in the right hemisphere. To restrict the search volume to white matter, white matter masks were extracted from FreeSurfer parcellation, eroded by 1 voxel, and warped to MNI space using the diffeomorphic mapping computed from QSDR reconstruction. Masks were then averaged across participants and thresholded at .9 to produce an average white matter mask. QA and GFA images were also smoothed with a $4 \mathrm{~mm}$ FWHM Gaussian kernel before group-level analysis.

\section{Statistical Analysis}

Statistical analysis was conducted using the general linear model (GLM) framework implemented in SPM8 (Wellcome Trust Department of Imaging Neuroscience, University College London). For both QA and GFA, we performed voxelwise multiple regression analyses with metacognitive accuracy scores and nuisance covariates for age and gender. Cluster-size tests were used to test for significant regions using a cluster-forming threshold of $p<.005$ and a cluster size threshold of $p<.05$ (FWE corrected). Because the assumption of uniform smoothness (stationarity) is violated for warped structural images, standard cluster-size tests under random field theory are not valid (Hayasaka, Phan, Liberzon, Worsley, \& Nichols, 2004; Worsley, Andermann, Koulis, MacDonald, \& Evans, 1999). We therefore applied a nonstationary cluster extent correction, in which clusters are adjusted according to local smoothness, using the parametric random field theory nonstationarity correction implemented in the NS toolbox (fmri.wfubmc.edu/cms/software\#NS). Accounting for nonstationarity is critical as not performing this correction can lead to invalid conclusions in analysis of structural images (Moorhead et al., 2005). Significant clusters are displayed on FSL's FMRIB58_FA_1mm standard template using MRIcro software (www.cabiatl.com/mricro/mricro/ mricro.html).

\section{RESULTS}

\section{Behavioral Results}

In a counterbalanced design, participants $(n=42)$ completed a perceptual discrimination task and verbal recognition memory task in which they made 2-choice discriminations and then rated their confidence in the accuracy of their responses on a trial-by-trial basis (Figure 1; McCurdy et al., 2013; Fleming et al., 2010). The perceptual task was performed at an individually determined threshold using a 2-up 1-down adaptive staircase procedure that results in a convergence on $70 \%$ accuracy at the limit for individual performance (Fleming et al., 2010; Levitt, 1971). Analysis revealed that performance accuracy was well controlled by the staircase for all participants $(M=0.707, S D=0.02$, range $=0.67-0.73$ ). Overall, performance on the memory task was good and had similar mean accuracy $(M=0.69$, $S D=0.08$, range $=0.58-0.93$ )

A linear mixed model with participant included as a random effect revealed that RT significantly predicted confidence at the trial level in both the perceptual decision task $(t=-34.75, p<.001 ;$ int $=3.76$, estimate $=$ $-0.40)$ and memory retrieval task $(t=-28.45, p<.001$; int $=4.39$, estimate $=-0.17)$, indicating that more confident decisions were associated with faster responses. Overall, mean confidence was higher in the memory retrieval task $(M=4.01, S D=0.59)$ compared with the perceptual decision task $(M=3.27, S D=0.96)[t(41)=$ $5.32, p<.001]$, which may be attributed to the relative difficulty of the perceptual task, which was performed at an individually determined perceptual threshold. Mean confidence level also showed a significant correlation within individuals across the two tasks, $r(42)=0.41, p<$ .01. Together these results replicate previous findings (Baird et al., 2013; Fleming, Dolan, et al., 2012; Song et al., 2011) and suggest that confidence level reflects both a task-independent general level of confidence particular to an individual as well as a task-dependent level of confidence an individual has toward performance on a particular cognitive task.

SDT (Green \& Swets, 1966) was used to quantify individual differences in metacognitive ability (Type II sensitivity); here quantified as the ability to accurately link confidence with performance (see Quantification of Metacognitive Ability). SDT enables computational approaches to the quantification of Type II sensitivity that is independent of the potential confounding influence of Type I sensitivity $\left(d^{\prime}\right)$ on the primary task. Analysis confirmed that metacognitive ability in both the perceptual decision task $\left(A_{\text {roc }}\right)$ and recognition memory task $\left(M_{\text {ratio }}\right)$ were uncorrelated with Type I performance $\left(A_{\text {roc }}: r(40)=\right.$ $\left.0.07, p=.67 ; M_{\text {ratio }}: r(40)=-0.29, p=.06\right)$. Additionally, orientation discrimination threshold in the perceptual task was uncorrelated with perceptual $A_{\text {roc }}, r(40)=-0.11, p=$ .49 , indicating that $A_{\text {roc }}$ estimates were not confounded with variance in perceptual acuity. SDT estimates of metacognitive ability were thus confirmed to be independent of variance in primary task performance, allowing for a direct comparison of metacognitive ability across process domains. Analysis revealed that metacognitive accuracy for perceptual decisions $\left(A_{\text {roc }}\right)$ and mnemonic judgments $\left(M_{\text {ratio }}\right)$ were uncorrelated across individuals, $r(40)=0.15, p=$ .34 , indicating an intraindividual dissociation in metacognitive ability across process domains (Figure 2). To ensure that this result was not an artifact of the fact that metacognitive ability for memory and perception were in different units ( $M_{\text {ratio }}$ and $A_{\text {roc }}$ ), we calculated $M_{\text {ratio }}$ for the 


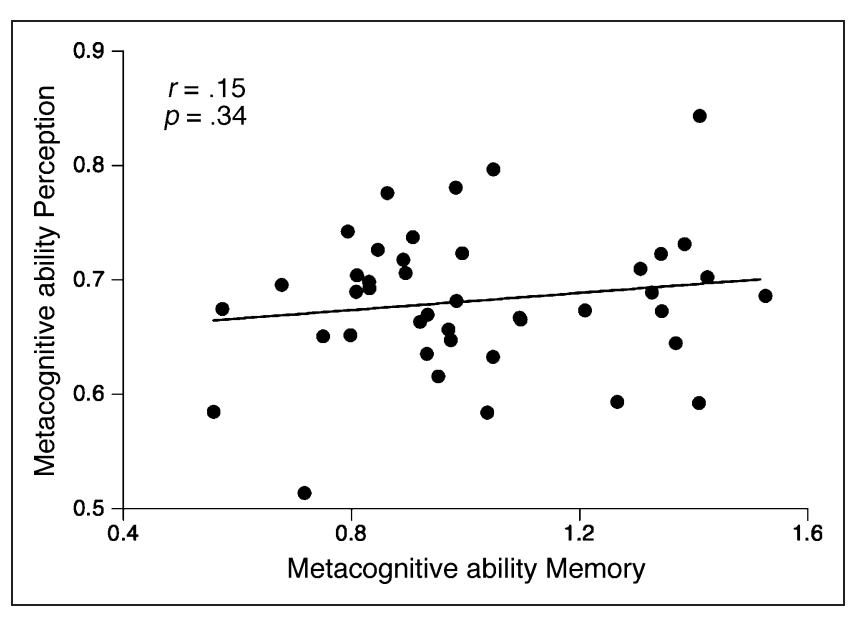

Figure 2. Scatterplot of zero-order correlation between metacognitive ability for perceptual decisions $\left(A_{\text {roc }}\right)$ and mnemonic judgments $\left(M_{\text {ratio }}\right)$ $[r(42)=0.15, p=.34]$.

perceptual discrimination task and correlated it with $M_{\text {ratio }}$ for the memory task. These measures were also uncorrelated across individuals, $r(40)=-0.07, p=.64$, indicating that the lack of correlation between perceptual and mnemonic metacognitive ability in our data cannot be attributed to differences in the computational approach or numerical scale between $M_{\text {ratio }}$ and $A_{\text {roc }}$. This result replicates two recent experiments using identical tasks and behavioral experimental designs (Baird, Mrazek, Phillips, \& Schooler, 2014; Baird et al., 2013). However, given that this finding is a null result, it nevertheless must be interpreted cautiously given the limited statistical power of each individual experiment. To increase the statistical power of this test, we therefore aggregated the data across these multiple studies to create a pooled sample of 135 participants. Integrated data analysis of this aggregated sample also revealed no correlation between metacognitive ability across memory and perception tasks, $r(133)=0.05, p=.57$.

\section{White Matter Microstructure and Tractography Results}

We next evaluated the relationship between white matter microstructure (diffusion anisotropy) and metacognitive ability for memory and perception. As shown in Figure 3A and Table 1, metacognitive accuracy for perceptual decisions $\left(A_{\text {roc }}\right)$ was associated with significantly increased GFA in the white matter underlying the right ACC $(p<$ .05 , FWE corrected; voxelwise threshold $p<.005)$. No suprathreshold clusters were observed between $A_{\text {roc }}$ and QA. As shown in Figure 3B and Table 1, metacognitive accuracy for memory retrieval $\left(M_{\text {ratio }}\right)$ was associated with increased QA in the white matter extending into the IPL in the region of the angular gyrus $(p<.05$, FWE corrected;

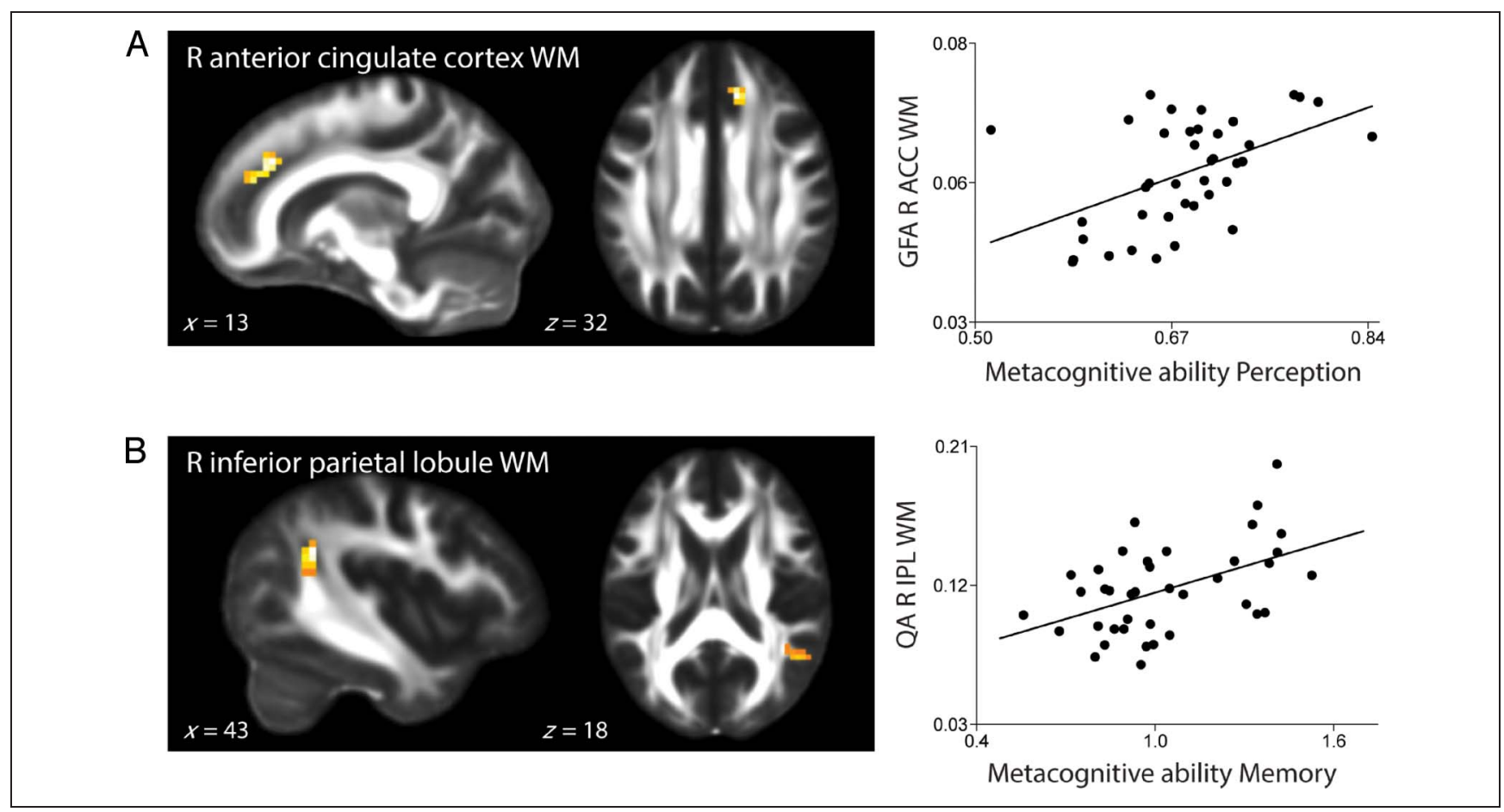

Figure 3. White matter microstructure (diffusion anisotropy) associated with metacognitive ability for memory and perception. (A) Metacognitive accuracy for perceptual decisions is associated with increased GFA in the white matter underlying the right ACC. (B) Metacognitive accuracy for memory retrieval is associated with increased QA in the white matter extending into the IPL. All clusters significant at $p<.05$, FWE corrected (height threshold, $p<.005$ ). Scatterplots show the correlations between metacognitive accuracy scores and median anisotropy values of significant clusters. $\mathrm{R}=$ right; $\mathrm{WM}=$ white matter. 
Table 1. White Matter Microstructure Associated with Metacognitive Ability in Memory and Perception Domains

\begin{tabular}{lcccccc}
\hline & Volume & \multicolumn{3}{c}{ Peak MNI } & \\
\cline { 5 - 6 } Region & $\left(\mathrm{mm}^{3}\right)$ & $X$ & $Y$ & $Z$ & $Z$ Value \\
\hline QA & & & & & \\
Memory $\left(M_{\text {ratio }}\right)$ & 93 & 42 & -48 & 27 & 3.85 \\
$\quad$ R IPL white matter & & & & & \\
Perception $\left(A_{\text {roc }}\right)$ & N/A & N/A & & & N/A \\
$\quad$ No suprathreshold cluster & & & & & \\
GFA & & & & & \\
Memory $\left(M_{\text {ratio }}\right)$ & & & & & \\
$\quad$ No suprathreshold cluster & N/A & N/A & & N/A \\
Perception $\left(A_{\text {roc }}\right)$ & & & & & \\
$\quad$ R ACC white matter & 78 & 12 & 30 & 33 & 3.48 \\
\hline
\end{tabular}

All clusters significant at $p<.05$, FWE corrected (height threshold, $p<.005)$.

voxelwise threshold $p<.005)$. No suprathreshold clusters were observed between $M_{\text {ratio }}$ and GFA. Additionally, no significant regions were observed linking variation in white matter microstructure in the left hemisphere to either metacognitive variable, and no significant regions were observed in which white matter microstructure negatively correlated with metacognitive ability in either domain.

We followed up this analysis by examining the anatomical connections of IPL and ACC white matter regions. For each cluster, we collected all streamlines that passed through the cluster, grouping the tracts according to the cortical regions they connected (see DSI Data Processing). We considered pairs of regions to be connected through the cluster if greater than $70 \%$ of the sample had streamlines connecting these regions passing through the cluster. As shown in Figure 4A, results revealed that ACC white matter cluster connected three pairs of regions: right anterior superior frontal gyrus (aSFG) to the right caudal ACC, right aSFG to left aSFG, and right aSFG to left caudal ACC. As shown in Figure 4B, the IPL white matter cluster connected 10 pairs of regions: right IPL to right caudal middle frontal gyrus (MFG), right IPL to right precentral gyrus, right IPL to right postcentral gyrus, a withinarea right IPL connection to right supramarginal gyrus, right IPL to right inferior temporal gyrus, right IPL to middle temporal gyrus, right IPL to the banks of the STS, right precentral gyrus to right inferior temporal gyrus, right precentral gyrus to right middle temporal gyrus, and right supramarginal gyrus to right middle temporal gyrus.

\section{DISCUSSION}

Replicating our previous studies (Baird et al., 2013, 2014), we found that the capacity of an individual to make accurate metacognitive evaluations of perceptual decisions and memory were uncorrelated, indicating an intraindividual dissociation in metacognitive ability across domains. This finding bolsters previous evidence for the notion that metacognitive skill in one domain may not necessarily translate to another (David et al., 2012; Metcalfe et al., 2012; Pannu \& Kaszniak, 2005; Schnyer et al., 2004). Furthermore, our results indicate that metacognitive accuracy in each domain was related to regional differences of white matter microstructure. Metacognitive ability in the perceptual domain was associated with increased
Figure 4. Tractography of ACC and IPL white matter regions in a representative subject. (A) The right ACC white matter cluster connected the right aSTG to the right caudal ACC (orange/red), right aSTG to left aSTG (turquoise), and right aSTG to left caudal ACC (purple). (B) The right IPL white matter cluster contained prominent tracts connecting IPL to MFG (orange/red), IPL to precentral gyrus (yellow), IPL to postcentral gyrus (green), IPL to superior/middle/inferior temporal lobe (purple), and within-area connections in IPL (dark blue) and supramarginal gyrus (light blue). Images are displayed in radiological convention: The left side of the brain reflects the right hemisphere.

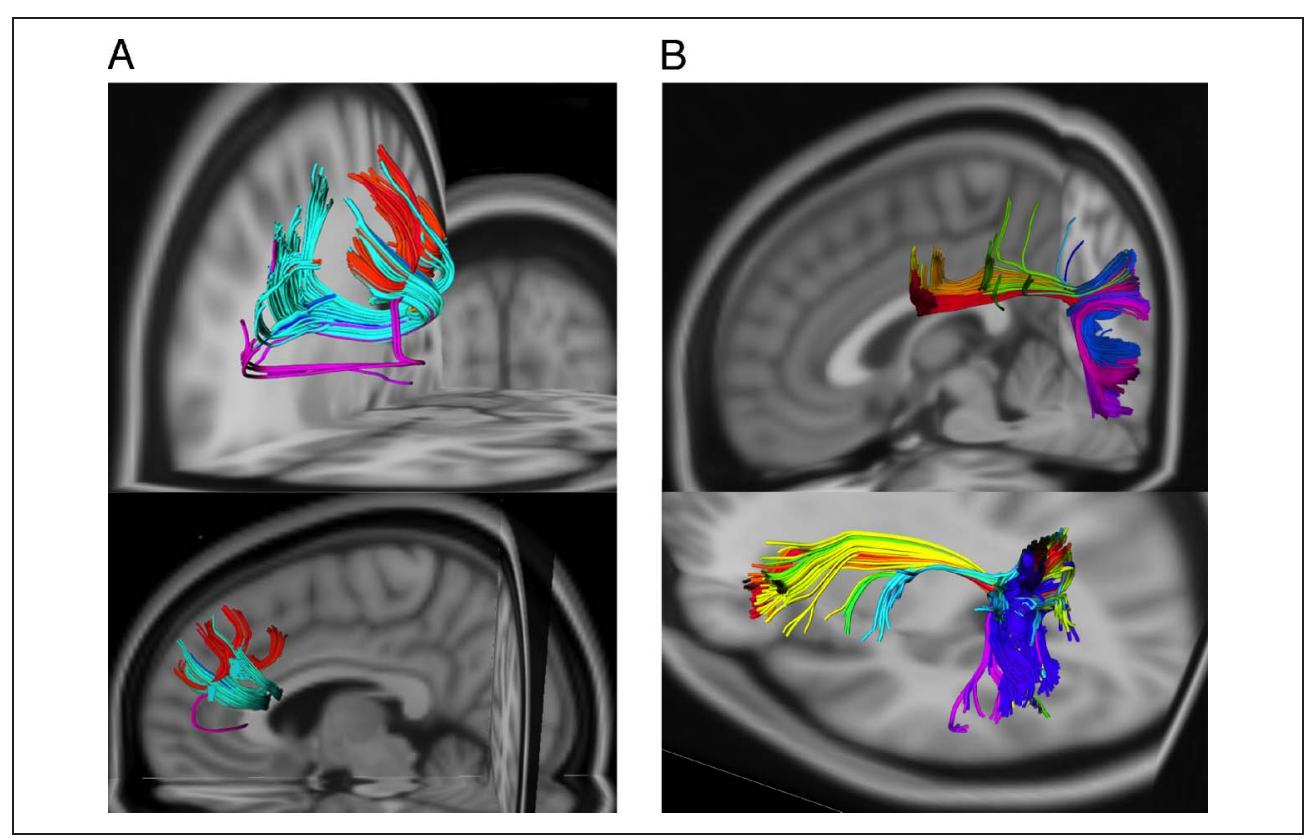


GFA in the white matter underlying the right ACC, whereas metacognitive ability in the memory domain was associated with increased QA in the white matter extending into the right IPL. ${ }^{1}$ Together, these results extend previous findings linking metacognitive ability in the domains of perception and memory to differences in distinct gray matter volume (McCurdy et al., 2013) and resting-state functional connectivity (Baird et al., 2013).

Tractography analysis of the right ACC white matter cluster associated with increased metacognitive ability on the perceptual task revealed that this region connected right aPFC (specifically the aSFG) to the right caudal ACC, right aPFC to left aPFC, and right aPFC to left caudal ACC. These results overlap with a recent connectivity-based parcellation of the human cingulate cortex, which revealed that this region has prominent anatomical connections to lateral aPFC, as well as the dorsal striatum (caudate nucleus and putamen; Beckmann, Johansen-Berg, \& Rushworth, 2009). The finding that metacognitive ability on the perceptual discrimination task was linked to increased white matter anisotropy underlying ACC therefore provides convergent evidence with our recent finding that metacognitive accuracy in this same task is associated with increased resting-state functional connectivity between lateral aPFC and the dACC and dorsal striatum (Baird et al., 2013). This finding also converges with other work linking metacognitive ability in the perceptual domain to the anatomically adjacent dACC, particularly the observation that lateral aPFC and dACC show increased activation during metacognitive assessments of visual discriminations and that the strength of activation in these regions during metacognitive judgments correlates with reported confidence (Fleming, Dolan, et al., 2012; Fleming, Huijgen, et al., 2012). A previous DTI study also found a positive association between perceptual metacognitive ability and FA in the anterior callosum linking left and right aPFC (Fleming et al., 2010). Although we did not observe a direct relationship between metacognitive ability and diffusion anisotropy in the anterior callosum, our tractography analysis revealed that the significant white matter cluster observed in our study contains fibers that pass through the anterior callosum connecting left and right aPFC, consistent with this previous result.

In the memory domain, we found that the ability to make accurate metacognitive judgments was associated with increased diffusion anisotropy in tracts extending into right IPL in the region near the angular gyrus. This finding is consistent with a broad range of studies documenting a primary role of IPL in meta-memory (e.g., Elman, Klostermann, Marian, Verstaen, \& Shimamura, 2012; Chua, Schacter, \& Sperling, 2009; Chua, Schacter, RandGiovannetti, \& Sperling, 2006). For instance, greater activity in a network including anterior prefrontal, mid/posterior cingulate, and lateral parietal regions is observed during memory monitoring in both feeling-of-knowing and retrospective confidence tasks (e.g., Chua et al., 2006, 2009). Moreover, IPL also shows greater activation for high- confidence hits in meta-memory tasks (Kim \& Cabeza, 2007; Wheeler \& Buckner, 2004) as well as strong "feelingof-knowing" judgments for semantic and episodic information (Elman et al., 2012). Finally, patients with parietal lesions produce fewer high-confidence recognition responses during retrieval (Simons, Peers, Mazuz, Berryhill, \& Olson, 2010; Davidson et al., 2008). This finding is also consistent with our recent observation that metacognitive ability for memory is associated with increased functional connectivity in a network including medial aPFC, MFG, and IPL (Baird et al., 2013). Tractography analysis of the significant IPL white matter cluster revealed that it contained prominent anatomical tracts connecting inferior parietal regions to the MFG, temporal lobe, and precentral gyrus, indicating a partial overlap in anatomical and functional networks underlying mnemonic metacognitive skill.

Altogether, the current findings converge with previous results in support of the proposal that an individual's capacity to accurately reflect on their cognitive processes is at least partially dependent on the type of cognitive process they are reflecting upon. Within this context, one possibility is that metacognitive evaluations in perceptual discrimination tasks primarily involve the capacity to monitor active representations. This type of metacognition may be best conceived of as an online monitor that integrates information over short timescales and may be linked to the related construct of cognitive control (Fleming \& Dolan, 2012; Fernandez-Duque, Baird, \& Posner, 2000). Indeed, white matter microstructure in the region of the anterior cingulate has also been linked to cognitive control (Metzler-Baddeley et al., 2012), and meta-analysis of functional imaging studies indicates that the anatomically adjacent dACC supports key control functions such as conflict and error detection (Beckmann et al., 2009; see Ridderinkhof, Ullsperger, Crone, \& Nieuwenhuis, 2004 , for a review). If metacognitive ability in perceptual discrimination tasks involves the accessibility of performance monitoring information in dACC to a wider network (Baird et al., 2013; Fleming et al., 2012), then microstructure in this region should play a key role. The current results are therefore consistent with a hypothesis put forward by Fleming and Dolan (2012) and Fleming et al. (2012) that metacognitive assessment of perceptual discriminations depends on the accessibility of information pertaining to the monitoring of immediate decisions (including errors and conflict, encoded in regions such as the dACC) to aPFC, which governs the transfer that information to a global frame of reference for metacognitive report.

In contrast, accurate metacognitive evaluations of memory may be understood to involve an appraisal of information pertaining to the content of memory, such as assessing the strength of a memory trace (Nelson \& Narens, 1990). As noted above, functional imaging studies of recognition memory and metamemory frequently observe activation in IPL alongside activation in the medial-temporal lobe across a wide array of stimuli and 
test conditions (e.g., Elman et al., 2012; Chua et al., 2006, 2009; Kim \& Cabeza, 2007; Wheeler \& Buckner, 2004). Although specifying the precise function of this region is a topic of active research, at least four different theories all implicate IPL in some form of coding of information pertaining to memory or in directing attention to memory representations (for reviews, see Olson \& Berryhill, 2009; Cabeza, Ciaramelli, Olson, \& Moscovitch, 2008; Wagner, Shannon, Kahn, \& Buckner, 2005). For instance, IPL has been proposed to serve as an accumulator for the strength of evidence for or against a memory decision (Wagner et al., 2005) to dynamically represent retrieved information as an output buffer (Vilberg \& Rugg, 2008; Vilberg, Moosavi, \& Rugg, 2006; Baddeley, 2000) or to support the subjective experience of the vividness of memories (Ally, Simons, McKeever, Peers, \& Budson, 2008). Accordingly, the finding that increased anisotropy in the white matter extending into IPL underlies enhanced metacognitive ability for memory may reflect the accessibility of memory information in IPL in the form of buffered episodic information, a memory strength signal, or memory vividness. Further elucidating the functional significance of this finding will be an important topic for future research.

As noted above, the finding that metacognitive ability across mnemonic and perceptual tasks did not correlate across individuals replicates two recent experiments using identical tasks and behavioral experimental designs (Baird et al., 2013, 2014). Furthermore, integrative data analysis of these studies combined with the current data indicates that the aggregated sample of participants from these studies ( $n=135$ ) also revealed no correlation between metacognitive ability across domains. Although we think that these results are clear and convincing, we nevertheless note that drawing firm conclusions regarding the behavioral stability of metacognitive accuracy across cognitive domains at the present time would still be premature. Aside from the current results and the two studies noted above, which all used identical tasks, only one other study (McCurdy et al., 2013) has compared metacognitive ability for perceptual and mnemonic judgments within individuals. As discussed above, the voxelbased morphometry findings of McCurdy et al. converge with Baird et al. (2013) and the current study in suggesting that individual differences in metacognitive ability for perception and memory relate to distinct features of brain architecture, and offer mutual support for some of the primary candidate brain regions. However, despite the dissociation at the neural level, McCurdy et al. reported a positive correlation between behavioral scores for perceptual and mnemonic metacognitive ability. One possibility is that this discrepancy could be attributed to differences between the tasks. For example, the memory task used in the current experiments involved a longer retention interval than the task used in McCurdy et al. Additionally, the memory task used in McCurdy et al. was a 2-alternative forced-choice (2AFC) task whereas our experiments have employed a 2-choice old/new discrimi- nation task, and there are differences between these two types of memory tests in the recruitment of recollection (Cook, Marsh, \& Hicks, 2005). Therefore, whereas McCurdy et al. employed a $2 \mathrm{AFC}$ design for both tasks, the current study used a 2AFC task for the perceptual task and a 2-choice old/new discrimination task for the memory task. Whether or how this difference in task structure across cognitive domains impacts individual performance and thus the stability of metacognitive accuracy across domains remains unclear. It will therefore be important for future research to examine the effect of manipulating the type and uncertainty of the Type I discrimination to observe whether there are some circumstances that are more conducive to observing a generalized metacognitive ability.

Additionally, although the present findings and those of Baird et al. (2013) and McCurdy et al. (2013) call into question a strict homogeneity of metacognition at the neural level, it is important to bear in mind that the individual differences approach used in these studies identifies differences that underlie the capacity for accurate metacognitive assessment rather than a comprehensive account of the neural processes that contribute to metacognitive judgments. The regional specificity observed in white matter microstructure, gray matter volume and functional connectivity identified across these studies should therefore primarily be regarded to reflect crosssectional differences that underlie the capacity for accurate metacognition, rather than an exhaustive account of the neural processes that contribute to metacognitive judgments in either domain. Indeed, it remains plausible that some domain-general regions may be recruited across different types of metacognitive tasks, a possibility that is supported by task-based comparisons of confidence judgments in memory and perception tasks using fMRI (Fleck, Daselaar, Dobbins, \& Cabeza, 2006).

In conclusion, the current findings demonstrate that the ability to make accurate metacognitive evaluations in perceptual and mnemonic domains relate to regional differences of white matter microstructure and lend support to the recent finding that metacognitive ability in each of these domains is linked to the strength of functional coupling within distinct cortical networks (Baird et al., 2013). These findings also illustrate how the investigation of white matter structure with high-resolution DSI can capture anatomical variation in white matter connection strengths (which may be inaccessible to other techniques) that are important to higher-order cognitive functions.

\section{Acknowledgments}

We thank Philip Beach, Mario Mendoza, Michael Mrazek, and Benjamin Mooneyham for assistance in conducting the research. We acknowledge support from the Center for Scientific Computing at the CNSI and MRL: NSF MRSEC (DMR-1121053) and NSF CNS-0960316. B. B. was supported by a National Science Foundation Graduate Research Fellowship under Grant DGE0707430. This research was supported by a grant from the U.S. 
Department of Education (Grant R305A110277) awarded to J. W. S. The content of this article does not necessarily reflect the position or policy of the U.S. Government, and no official endorsement should be inferred.

Reprint requests should be sent to Benjamin Baird, Department of Psychological and Brain Sciences, University of California, Santa Barbara, CA 93106-9660, or via e-mail: baird@psych. ucsb.edu.

\section{Note}

1. The finding that metacognitive ability on each task was related differentially to QA and GFA is not fully understood, and this differential effect was unexpected. At the current time, there is insufficient knowledge about the underlying tissue structure to predict when they will provide the same or different results. At this point, they are complementary techniques. Furthermore, given that GFA and QA are susceptible to different sources of MR noise (i.e., receiver gain or B1 inhomogeneity), we cannot rule out that the differential effect on GFA and QA may be related to differences in the sensitivity of these measures across different brain regions (Yeh, Verstynen, et al., 2013).

\section{REFERENCES}

Alexander, A. L., Hasan, K. M., Lazar, M., Tsuruda, J. S., \& Parker, D. L. (2001). Analysis of partial volume effects in diffusion-tensor MRI. Magnetic Resonance in Medicine, 45, 770-780

Ally, B. A., Simons, J. S., McKeever, J. D., Peers, P. V., \& Budson, A. E. (2008). Parietal contributions to recollection: Electrophysiological evidence from aging and patients with parietal lesions. Neuropsychologia, 46, 1800-1812.

Ashburner, J., \& Friston, K. J. (1999). Nonlinear spatial normalization using basis functions. Human Brain Mapping, 7, 254-266.

Baddeley, A. (2000). The episodic buffer: A new component of working memory? Trends in Cognitive Sciences, 4, 417-423.

Baird, B., Mrazek, M., Phillips, D. T., \& Schooler, J. W. (2014). Domain-specific enhancement of metacognitive ability following meditation training. Journal of Experimental Psychology: General, 143, 1972-1979.

Baird, B., Smallwood, J., Gorgolewski, K. J., \& Margulies, D. S. (2013). Medial and lateral networks in anterior prefrontal cortex support metacognitive ability for memory and perception. The Journal of Neuroscience, 33, 16657-16665.

Barrick, T. R., \& Clark, C. A. (2004). Singularities in diffusion tensor fields and their relevance in white matter fiber tractography. Neuroimage, 22, 481-491.

Beckmann, M., Johansen-Berg, H., \& Rushworth, M. F. (2009). Connectivity-based parcellation of human cingulate cortex and its relation to functional specialization. The Journal of Neuroscience, 29, 1175-1190.

Brainard, D. H. (1997). The Psychophysics Toolbox. Spatial Vision, 10, 433-436

Cabeza, R., Ciaramelli, E., Olson, I. R., \& Moscovitch, M. (2008). The parietal cortex and episodic memory: An attentional account. Nature Reviews Neuroscience, 9, 613-625.

Christoff, K., Gordon, A. M., Smallwood, J., Smith, R., \& Schooler, J. W. (2009). Experience sampling during fMRI reveals default network and executive system contributions to mind wandering. Proceedings of the National Academy of Sciences, 106, 8719-8724.
Chua, E. F., Schacter, D. L., Rand-Giovannetti, E., \& Sperling, R. A. (2006). Understanding metamemory: Neural correlates of the cognitive process and subjective level of confidence in recognition memory. Neuroimage, 29, $1150-1160$

Chua, E. F., Schacter, D. L., \& Sperling, R. A. (2009). Neural correlates of metamemory: A comparison of feeling-ofknowing and retrospective confidence judgments. Journal of Cognitive Neuroscience, 21, 1751-1765.

Cieslak, M., \& Grafton, S. T. (2014). Local termination pattern analysis: A tool for comparing white matter morphology. Brain Imaging and Behavior, 8, 292-299.

Clarke, F. R., Birdsall, T. G., \& Tanner, W. (1959). Two types of ROC curves and definitions of parameters. The Journal of the Acoustical Society of America, 31, 629-630.

Cohen-Adad, J., Descoteaux, M., \& Wald, L. L. (2011). Quality assessment of high angular resolution diffusion imaging data using bootstrap on Q-ball reconstruction. Journal of Magnetic Resonance Imaging, 33, 1194-1208.

Cook, G. I., Marsh, R. L., \& Hicks, J. L. (2005). Revisiting the role of recollection in item versus forced-choice recognition memory. Psychonomic Bulletin and Review, 12, 720-725.

Dale, A. M., Fischl, B., \& Sereno, M. I. (1999). Cortical surfacebased analysis. I. Segmentation and surface reconstruction. Neuroimage, 9, 179-194.

David, A. S., Bedford, N., Wiffen, B., \& Gilleen, J. (2012). Failures of metacognition and lack of insight in neuropsychiatric disorders. Philosophical Transactions of the Royal Society, Series B, Biological Sciences, 367, 1379-1390.

Davidson, P. S. R., Anaki, D., Ciaramelli, E., Cohn, M., Kim, A. S. N., Murphy, K. J., et al. (2008). Does lateral parietal cortex support episodic memory?: Evidence from focal lesion patients. Neuropsychologia, 46, 1743-1755.

Elman, J. A., Klostermann, E. C., Marian, D. E., Verstaen, A., \& Shimamura, A. P. (2012). Neural correlates of metacognitive monitoring during episodic and semantic retrieval. Cognitive, Affective \& Behavioral Neuroscience, 12, 599-609.

Fernandez-Duque, D., Baird, J. A., \& Posner, M. I. (2000). Executive attention and metacognitive regulation. Consciousness and Cognition, 9, 288-307.

Fischl, B., \& Dale, A. M. (2000). Measuring the thickness of the human cerebral cortex from magnetic resonance images. Proceedings of the National Academy of Sciences, U.S.A. 97, 11050-11055.

Fischl, B., Liu, A., \& Dale, A. M. (2001). Automated manifold surgery: Constructing geometrically accurate and topologically correct models of the human cerebral cortex. IEEE Transactions on Medical Imaging, 20, 70-80.

Fischl, B., Salat, D. H., Busa, E., Albert, M., Dieterich, M., Haselgrove, C., et al. (2002). Whole brain segmentation: Automated labeling of neuroanatomical structures in the human brain. Neuron, 33, 341-355.

Fischl, B., Salat, D. H., van der Kouwe, A. J., Makris, N., Segonne, F., Quinn, B. T., et al. (2004). Sequenceindependent segmentation of magnetic resonance images. Neuroimage, 23(Suppl. 1), S69-S84.

Fischl, B., Sereno, M. I., \& Dale, A. M. (1999). Cortical surfacebased analysis. II: Inflation, flattening, and a surface-based coordinate system. Neuroimage, 9, 195-207.

Fischl, B., Sereno, M. I., Tootell, R. B., \& Dale, A. M. (1999). High-resolution intersubject averaging and a coordinate system for the cortical surface. Human Brain Mapping, 8, 272-284

Fischl, B., van der Kouwe, A., Destrieux, C., Halgren, E., Segonne, F., Salat, D. H., et al. (2004). Automatically parcellating the human cerebral cortex. Cerebral Cortex, 14, 11-22. 
Fleck, M. S., Daselaar, S. M., Dobbins, I. G., \& Cabeza, R. (2006). Role of prefrontal and anterior cingulate regions in decisionmaking processes shared by memory and nonmemory tasks. Cerebral Cortex, 16, 1623-1630.

Fleming, S. M., \& Dolan, R. J. (2012). The neural basis of metacognitive ability. Philosophical Transactions of the Royal Society of London, Series B, Biological Sciences, 367, 1338-1349.

Fleming, S. M., Dolan, R. J., \& Frith, C. D. (2012). Metacognition: Computation, biology and function. Philosophical Transactions of the Royal Society, Series B, Biological Sciences, 367, 1280-1286.

Fleming, S. M., Huijgen, J., \& Dolan, R. J. (2012). Prefrontal contributions to metacognition in perceptual decision making. Journal of Neuroscience, 32, 6117-6125.

Fleming, S. M., \& Lau, H. C. (2014). How to measure metacognition. Frontiers in Human Neuroscience, 8, 443.

Fleming, S. M., Weil, R. S., Nagy, Z., Dolan, R. J., \& Rees, G. (2010). Relating introspective accuracy to individual differences in brain structure. Science, 329, 1541-1543.

Fritzsche, K. H., Laun, F. B., Meinzer, H.-P., \& Stieltjes, B. (2010). Opportunities and pitfalls in the quantification of fiber integrity: What can we gain from Q-ball imaging? Neuroimage, 51, 242-251.

Galvin, S. J., Podd, J. V., Drga, V., \& Whitmore, J. (2003). Type 2 tasks in the theory of signal detectability: Discrimination between correct and incorrect decisions. Psychonomic Bulletin \& Review, 10, 843-876.

Gazzaniga, M. S., \& LeDoux, J. E. (1978). The integrated mind. New York: Plenum.

Green, D. M., \& Swets, J. A. (1966). Signal detection theory and psychophysics. New York: Wiley.

Greve, D. N., \& Fischl, B. (2009). Accurate and robust brain image alignment using boundary-based registration. Neuroimage, 48, 63.

Hagmann, P., Cammoun, L., Gigandet, X., Meuli, R., Honey, C. J., Wedeen, V. J., et al. (2008). Mapping the structural core of human cerebral cortex. PLoS Biology, 6, e159.

Hall, L., Johansson, P., Tärning, B., Sikström, S., \& Deutgen, T. (2010). Magic at the marketplace: Choice blindness for the taste of jam and the smell of tea. Cognition, 117, 54-61.

Han, X., Jovicich, J., Salat, D., van der Kouwe, A., Quinn, B., Czanner, S., et al. (2006). Reliability of MRI-derived measurements of human cerebral cortical thickness: The effects of field strength, scanner upgrade and manufacturer. Neuroimage, 32, 180-194.

Hayasaka, S., Phan, K. L., Liberzon, I., Worsley, K. J., \& Nichols, T. E. (2004). Nonstationary cluster-size inference with random field and permutation methods. Neuroimage, 22, 676-687.

Higham, P. A., Perfect, T. J., \& Bruno, D. (2009). Investigating strength and frequency effects in recognition memory using type-2 signal detection theory. Journal of Experimental Psychology: Learning, Memory, and Cognition, 35, 57.

Johansson, P., Hall, L., Sikström, S., \& Olsson, A. (2005). Failure to detect mismatches between intention and outcome in a simple decision task. Science, 310, 116-119.

Jones, D. K., Knösche, T. R., \& Turner, R. (2013). White matter integrity, fiber count, and other fallacies: The do's and don'ts of diffusion MRI. Neuroimage, 73, 239-254.

Jovicich, J., Czanner, S., Greve, D., Haley, E., van der Kouwe, A., Gollub, R., et al. (2006). Reliability in multi-site structural MRI studies: Effects of gradient non-linearity correction on phantom and human data. Neuroimage, 30, 436-443.

Kim, H., \& Cabeza, R. (2007). Trusting our memories: Dissociating the neural correlates of confidence in veridical versus illusory memories. The Journal of Neuroscience, 27 , 12190-12197.
Kleiner, M., Brainard, D., Pelli, D., Ingling, A., Murray, R., \& Broussard, C. (2007). What's new in Psychtoolbox-3. Perception, 36, 1.1-1.16.

Kornbrot, D. E. (2006). Signal detection theory, the approach of choice: Model-based and distribution-free measures and evaluation. Perception \& Psychophysics, 68, 393-414.

Kraus, M. F., Susmaras, T., Caughlin, B. P., Walker, C. J., Sweeney, J. A., \& Little, D. M. (2007). White matter integrity and cognition in chronic traumatic brain injury: A diffusion tensor imaging study. Brain, 130, 2508-2519.

Kubicki, M., Park, H., Westin, C., Nestor, P., Mulkern, R., Maier, S., et al. (2005). DTI and MTR abnormalities in schizophrenia: Analysis of white matter integrity. Neuroimage, 26, 1109-1118.

Levitt, H. (1971). Transformed up-down methods in psychoacoustics. The Journal of the Acoustical Society of America, 49, 2-467.

Maniscalco, B., \& Lau, H. (2012). A signal detection theoretic approach for estimating metacognitive sensitivity from confidence ratings. Consciousness and Cognition, 21, 422-430.

McCurdy, L. Y., Maniscalco, B., Metcalfe, J., Liu, K. Y., de Lange, F. P., \& Lau, H. (2013). Anatomical coupling between distinct metacognitive systems for memory and visual perception. The Journal of Neuroscience, 33, 1897-1906.

Metcalfe, J., Van Snellenberg, J. X., DeRosse, P., Balsam, P., \& Malhotra, A. K. (2012). Judgements of agency in schizophrenia: An impairment in autonoetic metacognition. Philosophical Transactions of the Royal Society, Series B, Biological Sciences, 367, 1391-1400.

Metcalfe, J. E., \& Shimamura, A. P. (1994). Metacognition: Knowing about knowing. Cambridge, MA: MIT Press.

Metzler-Baddeley, C., Jones, D. K., Steventon, J., Westacott, L., Aggleton, J. P., \& O’Sullivan, M. J. (2012). Cingulum microstructure predicts cognitive control in older age and mild cognitive impairment. The Journal of Neuroscience, 32, 17612-17619.

Mickes, L., Wixted, J. T., \& Wais, P. E. (2007). A direct test of the unequal-variance signal detection model of recognition memory. Psychonomic Bulletin \& Review, 14, 858-865.

Moorhead, T. W. J., Job, D. E., Spencer, M. D., Whalley, H. C., Johnstone, E. C., \& Lawrie, S. M. (2005). Empirical comparison of maximal voxel and non-isotropic adjusted cluster extent results in a voxel-based morphometry study of comorbid learning disability with schizophrenia. Neuroimage, 28, 544-552.

Nelson, T. O., \& Narens, L. (1990). Metamemory: A theoretical framework and new findings. The Psychology of Learning and Motivation, 26, 125-141.

Nisbett, R. E., \& Wilson, T. D. (1977). Telling more than we can know: Verbal reports on mental processes. Psychological Review, 84, 231.

Olson, I. R., \& Berryhill, M. (2009). Some surprising findings on the involvement of the parietal lobe in human memory. Neurobiology of Learning and Memory, 91, 155-165.

Oouchi, H., Yamada, K., Sakai, K., Kizu, O., Kubota, T., Ito, H., et al. (2007). Diffusion anisotropy measurement of brain white matter is affected by voxel size: Underestimation occurs in areas with crossing fibers. American Journal of Neuroradiology, 28, 1102-1106.

Overgaard, M., \& Sandberg, K. (2012). Kinds of access: Different methods for report reveal different kinds of metacognitive access. Philosophical Transactions of the Royal Society, Series B, Biological Sciences, 367, 1287-1296.

Pannu, J. K., \& Kaszniak, A. W. (2005). Metamemory experiments in neurological populations: A review. Neuropsychology Review, 15, 105-130. 
Ridderinkhof, K. R., Ullsperger, M., Crone, E. A., \& Nieuwenhuis, S. (2004). The role of the medial frontal cortex in cognitive control. Science Signaling, 306, 443.

Schmahmann, J. D., Pandya, D. N., Wang, R., Dai, G., D’Arceuil, H. E., de Crespigny, A. J., et al. (2007). Association fibre pathways of the brain: Parallel observations from diffusion spectrum imaging and autoradiography. Brain, 130, 630-653.

Schnyer, D. M., Verfaellie, M., Alexander, M. P., LaFleche, G., Nicholls, L., \& Kaszniak, A. W. (2004). A role for right medial prefrontal cortex in accurate feeling-of-knowing judgments: Evidence from patients with lesions to frontal cortex. Neuropsychologia, 42, 957-966.

Schooler, J. W., \& Schreiber, C. A. (2004). Experience, metaconsciousness, and the paradox of introspection. Journal of Consciousness Studies, 11, 7-8.

Schooler, J. W. (2002). Re-representing consciousness: Dissociations between experience and meta-consciousness. Trends in Cognitive Sciences, 6, 339-344.

Schooler, J. W., Reichle, E. D., \& Halpern, D. V. (2004). Zoning out while reading: Evidence for dissociations between experience and metaconsciousness. In D. T. Levin (Ed.), Thinking and seeing: Visual metacognition in adults and children (pp. 203-226). Cambridge, MA: MIT Press.

Schooler, J. W., Smallwood, J., Christoff, K., Handy, T. C., Reichle, E. D., \& Sayette, M. A. (2011). Meta-awareness, perceptual decoupling and the wandering mind. Trends in Cognitive Sciences, 15, 319-326.

Segonne, F., Dale, A. M., Busa, E., Glessner, M., Salat, D., Hahn, H. K., et al. (2004). A hybrid approach to the skull stripping problem in MRI. Neuroimage, 22, 1060-1075.

Shimamura, A. P. (2000). The role of the prefrontal cortex in dynamic filtering. Psychobiology, 28, 207-218.

Simons, J. S., Peers, P. V., Mazuz, Y. S., Berryhill, M. E., \& Olson, I. R. (2010). Dissociation between memory accuracy and memory confidence following bilateral parietal lesions. Cerebral Cortex, 20, 479-485.

Smallwood, J., McSpadden, M., \& Schooler, J. W. (2008). When attention matters: The curious incident of the wandering mind. Memory \& Cognition, 36, 1144-1150.

Song, C., Kanai, R., Fleming, S. M., Weil, R. S., Schwarzkopf, D. S., \& Rees, G. (2011). Relating inter-individual differences in metacognitive performance on different perceptual tasks. Consciousness and Cognition, 20, 1787-1792.

Swets, J. A. (1986). Form of empirical ROCs in discrimination and diagnostic tasks: Implications for theory and measurement of performance. Psychol Bull, 99, 181-198.
Terrace, H. S., \& Metcalf, J. S. (2004). The missing link in cognition: Origins of self-reflective consciousness. Oxford University Press.

Tuch, D. S. (2004). Q-ball imaging. Magnetic Resonance in Medicine, 52, 1358-1372.

Vilberg, K. L., \& Rugg, M. (2008). Memory retrieval and the parietal cortex: A review of evidence from a dual-process perspective. Neuropsychologia, 46, 1787.

Vilberg, K. L., Moosavi, R. F., \& Rugg, M. D. (2006). The relationship between electrophysiological correlates of recollection and amount of information retrieved. Brain Research, 1122, 161.

Vos, S. B., Jones, D. K., Viergever, M. A., \& Leemans, A. (2011). Partial volume effect as a hidden covariate in DTI analyses. Neuroimage, 55, 1566-1576.

Wagner, A. D., Shannon, B. J., Kahn, I., \& Buckner, R. L. (2005). Parietal lobe contributions to episodic memory retrieval. Trends in Cognitive Sciences, 9, 445-453.

Wedeen, V. J., Hagmann, P., Tseng, W. Y. I., Reese, T. G., \& Weisskoff, R. M. (2005). Mapping complex tissue architecture with diffusion spectrum magnetic resonance imaging. Magnetic Resonance in Medicine, 54, 1377-1386.

Wheeler, M. E., \& Buckner, R. L. (2004). Functional-anatomic correlates of remembering and knowing. Neuroimage, 21, $1337-1349$

Wilson, M. (1988). MRC Psycholinguistic Database: Machineusable dictionary, version 2.00. Behavior Research Methods, Instruments, \& Computers, 20, 6-10.

Worsley, K., Andermann, M., Koulis, T., MacDonald, D., \& Evans, A. (1999). Detecting changes in nonisotropic images. Human Brain Mapping, 8, 98-101.

Yeh, F. C., Tang, P. F., \& Tseng, W. Y. I. (2013). Diffusion MRI connectometry automatically reveals affected fiber pathways in individuals with chronic stroke. Neuroimage: Clinical, 2, 912-921.

Yeh, F.-C., \& Tseng, W. Y. I. (2011). NTU-90: A high angular resolution brain atlas constructed by q-space diffeomorphic reconstruction. Neuroimage, 58, 91-99.

Yeh, F.-C., Verstynen, T. D., Wang, Y., Fernández-Miranda, J. C., \& Tseng, W.-Y. I. (2013). Deterministic diffusion fiber tracking improved by quantitative anisotropy. PloS One, 8 , e80713.

Yeh, F.-C., Wedeen, V. J., \& Tseng, W.-Y. (2010). Generalizedsampling imaging. Medical Imaging, IEEE Transactions on, 29, 1626-1635. 